\title{
Affine Illumination compensation on hyperspectral/multiangular remote sensing images *
}

\author{
Pedro Latorre Carmona ${ }^{1}$, Luis Alonso ${ }^{2}$, Filiberto $\mathrm{Pla}^{1}$, Jose E. Moreno ${ }^{2}$, and Crystal \\ Schaaf $^{3}$ \\ 1 Dept. Lenguajes y Sistemas Informáticos, Jaume I University, Spain \\ 2 Departamento de Física de la Tierra y Termodinámica, Universidad de Valencia \\ 3 Department of Geography and Environment, Center for Remote Sensing, Boston University \\ [latorre,pla] @lsi.uji.es, luis.alonso@uv.es, jose.moreno@uv.es, schaaf@bu.edu
}

Keywords: Affine illumination compensation, hyperspectral/multiangular images

\begin{abstract}
The huge amount of information some of the new optical satellites developed nowadays will create demands to quickly and reliably compensate for changes in the atmospheric transmittance and varying solar illumination conditions. In this paper three different forms of affine transformation models (general, particular and diagonal) are considered as candidates for rapid compensation of illumination variations. They are tested on a group of three pairs of CHRISPROBA radiance images obtained in a test field in Barrax (Spain), and where there is a difference in the atmospheric as well as in the geometrical acquisition conditions. Results indicate that the proposed methodology is satisfactory for practical normalization of varying illumination and atmospheric conditions in remotely sensed images required for operational applications.
\end{abstract}

\section{Introduction}

Nowadays, there are satellites that are able to acquire images from the same site every day with a high spatial resolution (FORMOSAT -2). Other satellites with similar capabilities will be launched in the next future (like $S E N T I N E L-2$, [14]). A limiting factor in this series exploitation is the need to compensate for illumination effects due to the changing atmospheric transmittance conditions and solar illumination angles. Corrections are typically made using an atmospheric radiative transfer code. The problem, however, is typically the lack of information about the actual atmospheric status (water vapour, aerosols type, etc.). For some systems it is possible to derive this information from the acquired data itself, but this is not always the case (For the MERIS satellite, for instance, this is possible, but not for the SPOT satellite). An alternative would be to consider it from an illumination change assessment (normalization type compensation

\footnotetext{
* This work was supported by the Spanish Ministry of Science and Innovation under the projects Consolider Ingenio 2010CSD2007-00018, EODIX AY A2008 - 05965-C04-04/ESP and ALFI3DTIN2009 - $14103-C 03-01$, by the Generalitat Valenciana through the project PROMETEO/2010/028 and by Fundació Caixa-Castelló through the project $P 11 B 2007-48$.
} 
strategy) point of view, where the surface has not been altered, where only the scattering events in the atmosphere would be considered (the absorption affects would be removed by other methods). This approach is currently used in the MERIS/ENVISAT products [15]. A methodology following this strategy is presented in this paper, where the only information available should be an image taken as reference.

Healey et al theoretically proved [1], [2], that a change in the illumination conditions could be modeled by an affine transformation model. There exist three main different types of affine transformation models. The simplest one is given by a diagonal (matrix) transform of the feature space (diagonal model) [3]. This model, which corresponds to the so-called von-Kries adaptation in human colour vision [4], may be generalized considering a non-diagonal matrix transform (particular model) [5], and adding to this model an offset, i. e., a translation vector (general model) [6].

In this paper we analyze the applicability of the three different types of affine transformation models and compare their performance using a group of radiance images from the CHRIS-PROBA satellite acquired during July the $12^{\text {th }}$ and July the $14^{\text {th }}$ 2003 over Barrax (Spain). The combination CHRIS-PROBA provided multi-spectral and multi-angular images of this test site [8]. This paper is built on the research made by Latorre Carmona et al on atmospheric compensation (using affine compensation models) [17] for the case of synthetically radiance images generated using the $6 S$ code [7]. The organization of the paper is as follows: Section 2 introduces the three affine compensation models. Section 3 analyses the assumptions made in the paper with those made by the $6 S$ code for atmospheric correction. Section 4 presents the methodology used to register the images and presents and discusses the atmospheric compensation results obtained. Conclusions can be found in Section 5.

\section{Affine illumination compensation}

Assume a vector $\mathbf{x} \in \mathbb{R}_{+}^{D}$ representing a measurement from a $D$ bands linear multispectral sensor. The application of a transformation model is therefore valid whether $\mathbf{x}$ is considered as the radiance reaching the sensor, or the response of this sensor. Under a change in the illumination characteristics this vector will undergo a change $\mathrm{x} \rightarrow \widetilde{\mathbf{x}}$. The most general affine transformation model considers that both $\mathbf{x}, \widetilde{\mathbf{x}}$ are related through: $\widetilde{\mathbf{x}}=\mathbf{B} \cdot \mathbf{x}+\mathbf{t}$ [9]. In this equation, $\mathbf{B}$ is a $D \times D$ matrix and $\mathbf{t}$ is a $D \times 1$ vector. If the vector $\mathbf{t}$ is considered zero, the transformation model would be: $\widetilde{\mathbf{x}}=\mathbf{A} \cdot \mathbf{x}$, where $\mathbf{A}$ would also be a $D \times D$ matrix. This is the camera model considered by Healey et al [1]. Under certain conditions of the spectral response of the sensors [3], matrix A may be approximated as a diagonal matrix. The three models will be called hereafter general, particular and diagonal affine models.

One step in the application of the method to obtain the parameters of the general affine transformation model is the assessment of the inverse matrix of the matrix $\mathbf{F}$ obtained after the Cholesky decomposition of the covariance matrix of the data $(\mathrm{x})$. This assessment may present numerical instability problems due to some characteristics in the signal shape, as it can be the abrupt change in this shape in some specific spectral ranges (for instance, when dealing with the radiance coming from a vegetated surface). The inverse matrix can be obtained applying the Truncated Singular Value Decomposi- 
tion $(t-S V D)$ technique [12]. For more details about the methods used to apply the three affine models, see the Appendix A and [16], [9]. The $t-S V D$ technique can be found in the Appendix B.

\section{Comparison with standard atmospheric correction methods}

There are two main atmospheric processes to take into account, the gaseous absorption and the scattering by molecules and aerosols. In this paper, the main interest is in the scattering properties of the atmosphere. To isolate these changes from those of absorption, the peak stripping method [13] was applied, but for absorption valleys. The original method compares the value of channel $i$ with the mean of its 2 direct neighbours, i. e., $m_{i}=\frac{y_{(i-1)}+y_{(i+1)}}{2}$, and if $y_{i}<m_{i}$ then $y_{i} \leftarrow m_{i}$, and otherwise left unchanged. In our case, the condition is if $y_{i}>m_{i}$ then $y_{i} \leftarrow m_{i}$. This process is applied iteratively. Figure 1(a) shows the transmittance due to gases $\left(t_{g}\right)$ in the spectral interval $[400,1100] \mathrm{nm}$ simulated using the $6 S$ code for the $U S 62$ atmospheric model, with an $O_{3}$ (Ozone) content of 300 Dobson Units (DU) (i. e., $3 \mathrm{~cm}$ column) and the water vapour column at $2.5 \mathrm{~cm}$ (values close to those directly obtained in Barrax during the campaign made in June and July 2003 [8]). Figure 1(a) shows that main atmospheric absorption valleys due to gases appear in the wavelength region: $680 \leq \lambda \leq 1000 \mathrm{~nm}$. A technique to obtain a curve that may eliminate the atmospheric absorption valleys would just consist of normalizing the radiance curve per pixel by the $t_{g}$ curve. However, this curve must be found first, and this can only happen if we know the atmospheric composition at the time of acquisition or if it is modeled using a radiative transfer code like $6 S$. The advantage the method we apply has is that no prior knowledge about the atmosphere composition is necessary.

\section{Results and discussion}

A series of four images from CHRIS-PROBA were selected for the assessment of the three illumination compensation algorithms. These images were acquired in July the $12^{\text {th }}$ and July the $14^{\text {th }} 2003$ over Barrax (Spain). Image labeled $35 A 2$ was acquired in July the $12^{t h}$ 2003. It corresponds to a Flight Zenith Angle (FZA) $=0^{\circ}$. This image was selected as the reference image. Other three images obtained during July the $14^{t h}$ 2003 were considered as the images to be registered and compensated (called warp images) in relation to the reference one (35A2). Its FZA are $0^{\circ}$ (image labeled 3598), $+36^{\circ}$ (image labeled 3599) and $-36^{\circ}$ (image labeled $359 \mathrm{~A}$ ). In Figure $1(\mathrm{~b})$ a polar plot showing the image acquisition geometry for July the $12^{\text {th }}$ and the $14^{\text {th }}$ is shown. These images with a short time difference among them were selected in order to make sure all changes in the radiance came only from the illumination and geometry acquisition conditions, and not from changes in the surface (i. e., soil moisture or vegetation).

\subsection{Image registration}

Image $35 A 2$ was taken as reference. The rest of the images were co-registered in relation to this one, using Ground Control Points (GCPs) with sub-pixel precision. 100 

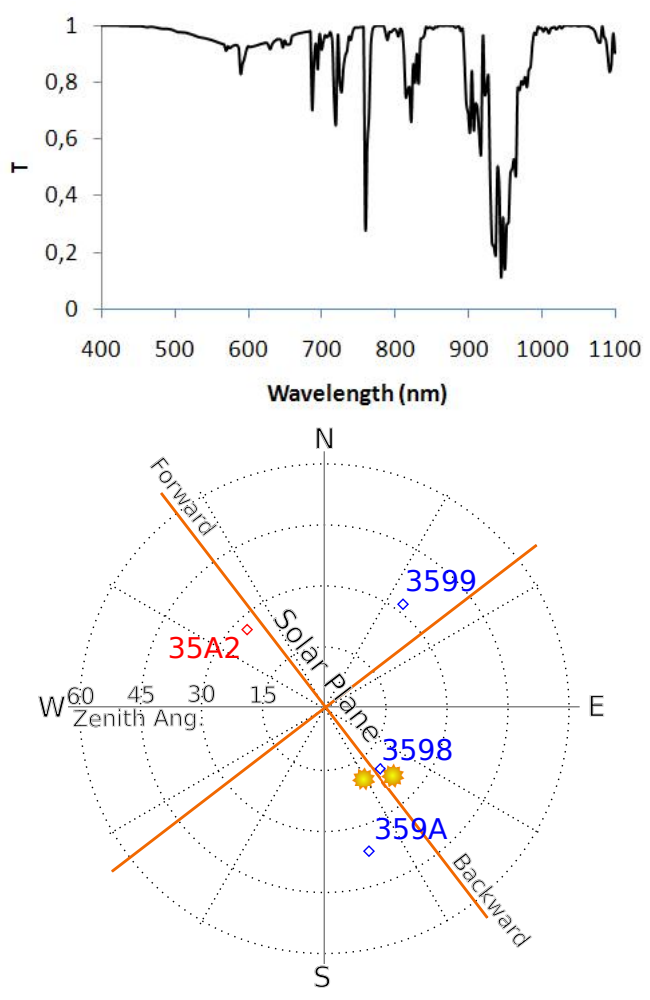

Fig. 1. (a) $t_{g}$ plot in the spectral interval $[400,1100] \mathrm{nm}$ simulated using the $6 S$ code using an atmospheric model called $U S 62$. (b) Polar plot of the acquisition geometry.

points were used for each image, with an $R M S E=0.3$ applying a $4^{\text {th }}$ order polynomial function. Re-sampling was made considering bi-cubic interpolation. A mask was created to remove some clouds (and the corresponding shadows) that were detected in the reference image. Other changes in the surface were included, like harvesting in some crops during the two days of difference between the images. Figure 2 shows a false colour $R G B$ image of $35 A 2,3598$, and the result of the registration.

\subsection{Scattering vs absorption}

Clayton method was applied with two restrictions: (a) fixing some specific wavelengths, so that the radiance for them was not updated on each iteration, (b) using two iteration values, 40 for wavelengths $\lambda \leq 751 \mathrm{~nm}$ and 120 for $\lambda>751 \mathrm{~nm}$, in order to preserve the chlorophyll activity region valley. A part of the wavelengths that were not updated were used to force the method not to smooth the chlorophyll absorption valley. The rest were selected as the local maxima for a radiance pixel of the terrain whose radiance curve were as flat and as smooth as possible. Figure 3(a),(b) shows the radiance curves corresponding to a pixel from a potato crop (dry barley in (b)) area of Barrax test 


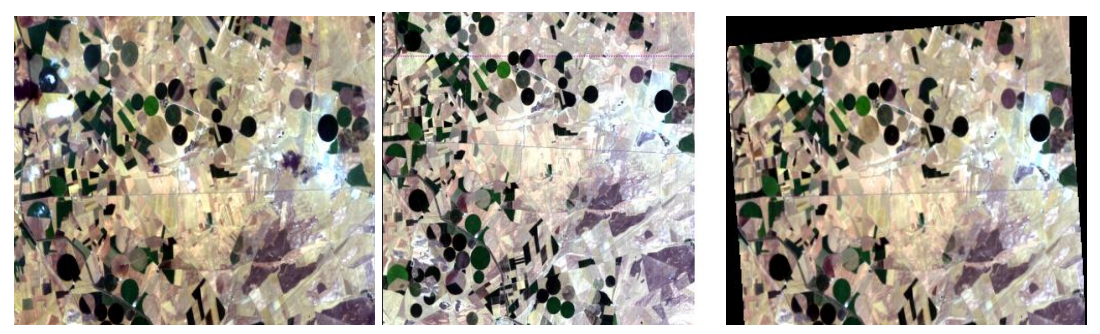

Fig. 2. 35A2, 3598, and registered images

site for the $35 A 2 / 3598$ image pair, before and after the application of the absorption valley removal strategy. The group of wavelengths $(583 \mathrm{~nm}, 605 \mathrm{~nm}, 674 \mathrm{~nm})$ were not updated during the application of the algorithm. Values higher than 30 for wavelengths lower than $751 \mathrm{~nm}$ created numerical instability problems in the assessment of the inverse matrix of the Cholesky decomposition of the covariance matrix of the pixel data, which is needed to apply the general affine compensation model. A Truncated Singular Value Decomposition Technique [12] was used to assess the inverse matrices. The value of 40 was considered as an intermediate value allowing the elimination of absorption peaks and conservation of the chlorophyll absorption valley, and the assessment of the corresponding inverse matrices.

\subsection{Illumination compensation}

Suppose two different point sets as $N \times D$ matrices (A and B), corresponding to two radiance images for two different illumination conditions. Considering also the case of compensating the illumination change of the second image, the aim would be to transform the second image $\mathbf{B} \rightarrow \widetilde{\mathbf{B}}$ into an image as close as possible to the first one. Thus, being the first image the target image, the following relative frobenius index could be established as a measure of the illumination compensation performance: $F_{I}=\frac{\|\mathbf{A}-\widetilde{\mathbf{B}}\|_{F}}{\|\mathbf{A}\|_{F}}$, where the Fobenius norm for a $N \times D$ matrix $\mathbf{X}$ is: $\|\mathbf{X}\|_{F}=\sqrt{\sum_{i=1}^{N} \sum_{j=1}^{D}\left|x_{i j}\right|^{2}}$. Table 1 shows the compensation capability of the three affine models, for each one of the pairs of images that were registered. The general and particular affine models are better than the diagonal model for the three pairs of images. The relative frobenius norm, before illumination compensation, for the case $35 A 2 / 3599$ pair, was particularly low. The highest frobenius norm before compensation was for the $35 A 2 / 3598$ pair. That may be due to the fact (see Figure 1(b)) that images $35 A 2$ and 3598 are almost aligned with the Sun, in the first case in opposition, and in the second case in conjunction. This geometry generates high angular effects on the surface reflectance. These effects are however minimized in the plane perpendicular to the Solar plane. Image 3599 is in that plane, whereas image $359 \mathrm{~A}$ is close to the principal plane. Therefore, difference between images before compensation are lowest in the $35 A 2 / 3599$ pair.

The capability of the three models when no information able to create masks is available during/after acquisition was also tested. Table 2 shows the compensation results for the case when all the pixels in the images were considered. As in the previous 

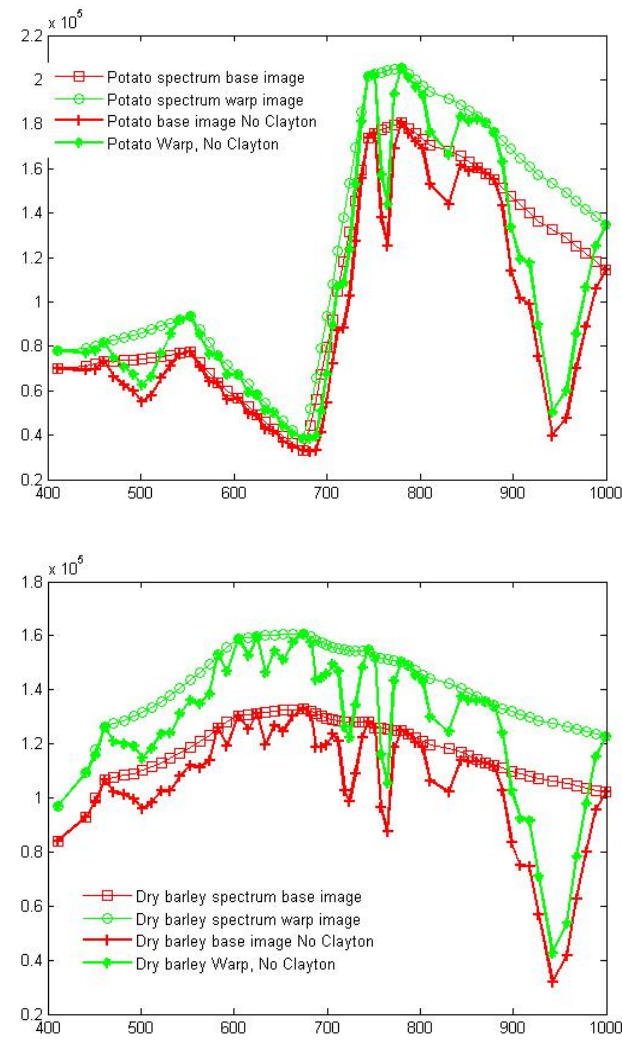

Fig. 3. Result of the application of the absorption valley removal strategy to the radiance curves of the reference and warp images for the case of a (a) potato crop pixel, (b) dry barley pixel, selected from the $35 A 2 / 3598$ CHRIS-PROBA image pair.

case, the relative frobenius norm, before illumination compensation, was low for the case $35 A 2 / 3599$ pair. The general affine model, for this pair gives a relative frobenius norm higher than for the case before compensation. That was caused by the fact that for a very small number of pixels the norm after compensation was higher than before compensation, but not for the rest of the image pixels.

Illumination compensation between images on a pixel by pixel basis was also tested. In this case, the ratio of the norm of the difference in the radiance vector between each pixel in the reference image and the pixel in the registered and compensated image, and the pixel in the reference image, was taken as the criteria. Figure 4 shows a colour coded image for this ratio for the $35 A 2 / 3598$ image pair. In all these cases, masks for clouds and shadows had been applied. There is a general tendency to the reduction in the difference between the images. However, there are some parts where this reduction is lower. That is the case for the two circular crops on top of all the images of Figure 4 , as well as for some small areas close to the pixels where a mask had been applied. 


\begin{tabular}{|c|c|c|c|c|}
\hline Pair & Before compensation & $\mathbf{x}^{\prime}=\mathbf{A} \cdot \mathbf{x}+\mathbf{t}$ & $\mathbf{x}^{\prime}=\mathbf{A} \cdot \mathbf{x}$ & $\mathbf{x}^{\prime}=\operatorname{diag}(\mathbf{A}) \cdot \mathbf{x}$ \\
\hline $35 A 2 / 3598$ & 0.214 & 0.059 & 0.056 & 0.062 \\
$35 A 2 / 359 A$ & 0.192 & 0.051 & 0.048 & 0.056 \\
$35 A 2 / 3599$ & 0.055 & 0.050 & 0.047 & 0.051 \\
\hline
\end{tabular}

Table 1. Compensation results for the three affine models

\begin{tabular}{|c|c|c|c|c|}
\hline Pair & Before compensation & $\mathbf{x}^{\prime}=\mathbf{A} \cdot \mathbf{x}+\mathbf{t}$ & $\mathbf{x}^{\prime}=\mathbf{A} \cdot \mathbf{x}$ & $\mathbf{x}^{\prime}=\operatorname{diag}(\mathbf{A}) \cdot \mathbf{x}$ \\
\hline $35 A 2 / 3598$ & 0.226 & 0.101 & 0.090 & 0.096 \\
$35 A 2 / 359 A$ & 0.206 & 0.093 & 0.086 & 0.092 \\
$35 A 2 / 3599$ & 0.091 & 0.092 & 0.084 & 0.088 \\
\hline
\end{tabular}

Table 2. Compensation results without applying the correction masks

In the case of the two circular crops, the difference could be attributed to a land-use change, and not to an illumination or acquisition geometry change. Figure 5 shows
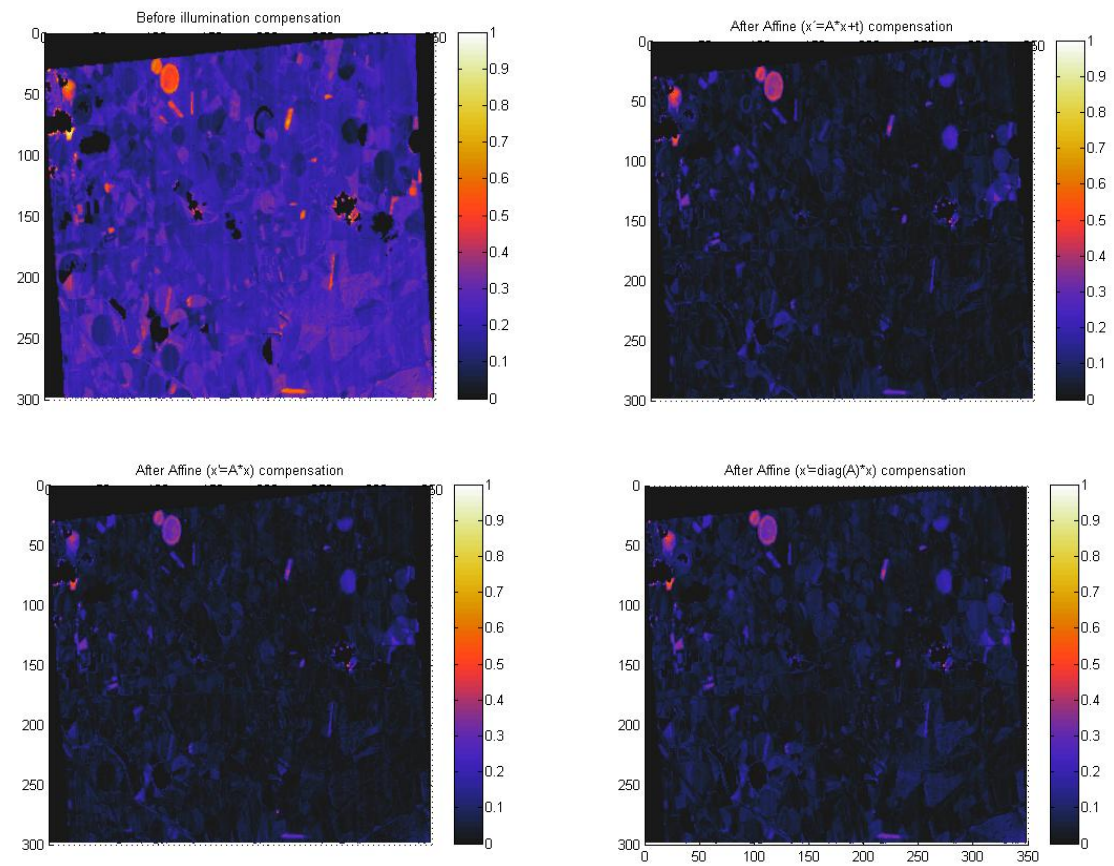

Fig. 4. Relative frobenius norm for the pair $35 A 2$ and 3598 (a) Before Compensation. (b) General affine compensation. (c) Particular affine compensation. (d) Diagonal affine compensation

the radiance curve for a group of four pixels for the reference image, for the image to be compensated, and for the resulting compensated image after the application of 
the general affine model. Three pixels corresponded to crops of different nature (corn, potato, and dry barley, Figure 5(a) to (c)). One pixel (Figure 5(d)) corresponded to one of the two circular crops with the highest difference after illumination compensation. In general terms, there is a small difference between the aim curve and the curve of the compensated image in each plot which proves the capacity of the method. However, last plot in Figure 5 shows that the general affine method is not able (nor the rest) to compensate for the difference in the radiance curves for that particular pixel (of one of the circular crops, please see Fig. 4(b)). This difference could be attributed to a surface change for which no previous information was available.
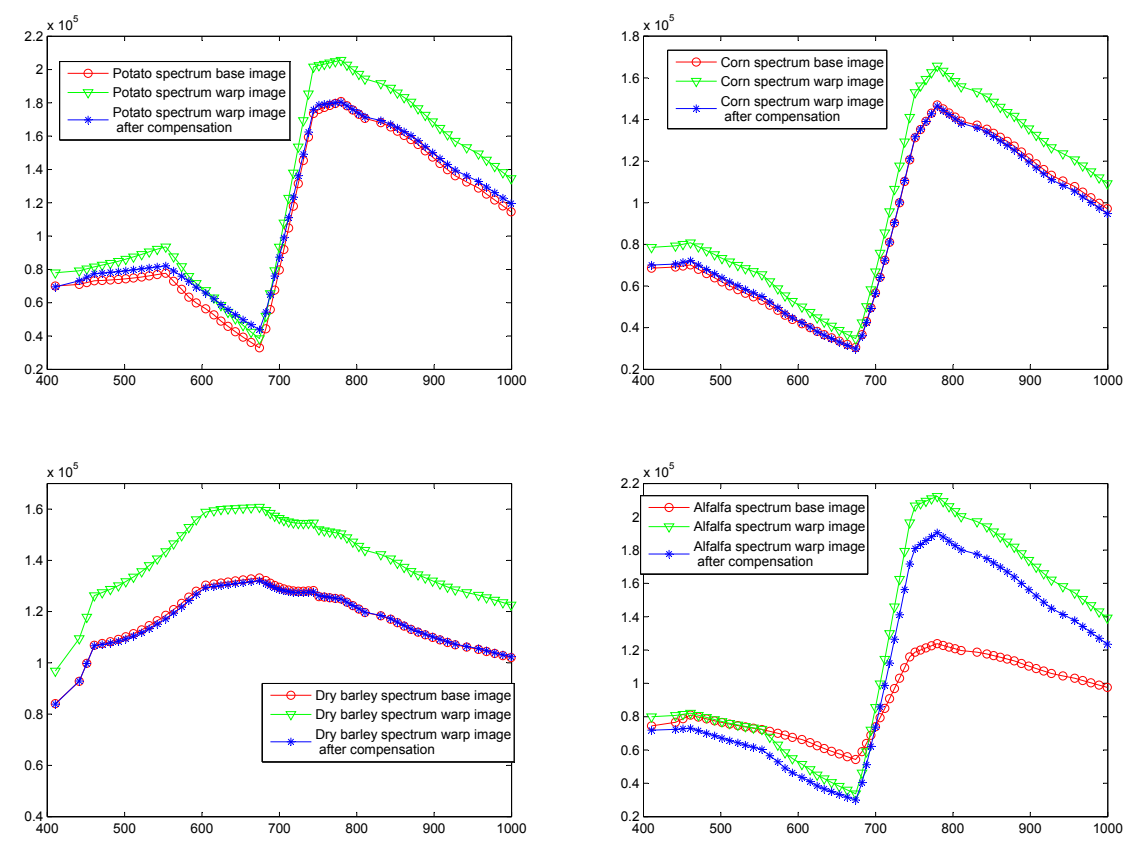

Fig. 5. Radiance curves of pixels of different crops for the reference, warp and warp after illumination compensation images

\section{Conclusions}

In this paper we have shown that the three affine compensation models (general, particular and diagonal) can be used to compensate for illumination variations in radiance images due to changes in the atmosphere and acquisition conditions, being the particular affine model the best of the three. The methodology presented in this paper is satisfactory for compensation of varying illumination and atmospheric conditions in remotely sensed images required for operational applications. 


\section{A Deduction of the illumination compensation formulae for the 3 affine models.}

Let $\mathbf{X}$ and $\widetilde{\mathbf{X}}$ be two $N \times D$ matrices representing two point sets, with $N$ the number of points in the set, and $\mathbf{C}$ and $\widetilde{\mathbf{C}}$ their covariance matrices. Applying the Cholesky Factorization to $\mathbf{C}$ and $\widetilde{\mathbf{C}}$, they can be written as: $\mathbf{C}=\mathbf{F} \cdot \mathbf{F}^{t}$ and $\widetilde{\mathbf{C}}=\widetilde{\mathbf{F}} \cdot \widetilde{\mathbf{F}}^{t}$, where $\mathbf{F}^{t}$ and $\widetilde{\mathbf{F}}^{t}$ are the transpose matrices of $\mathbf{F}$ and $\widetilde{\mathbf{F}}$ respectively. Points in the data set are first whitened (only shown for the first group), i. e. $\mathbf{y}=\mathbf{F}^{-1} \cdot \overline{\mathbf{x}}$, where $\overline{\mathbf{x}}=\mathbf{x}-\mathbb{E}\{\mathbf{x}\}$. This matrix may be ill-conditioned under some circumstances, and a technique like Truncated Singular Value Decomposition can be used to assess them. See Appendix B and [12] for details. Taking into account the previous equation, we have: $\mathbf{F} \cdot \widetilde{\mathbf{y}}=\mathbf{B} \cdot \mathbf{F} \cdot \mathbf{y}$, and creating a quadratic form of this last expression, the next expression follows: $\widetilde{\mathbf{F}} \cdot \widetilde{\mathbf{F}}^{t}=\mathbf{B} \cdot \mathbf{F} \cdot \mathbf{F}^{t} \cdot \mathbf{B}^{t}$. In [10], they proved that an equation of the form $\mathbf{T} \cdot \mathbf{T}^{t}=\mathbf{S} \cdot \mathbf{S}^{t}$ has a solution of the form $\mathbf{T}=\mathbf{S} \cdot \mathbf{P}$, where $\mathbf{P}$ is an orthonormal matrix. This will help finding the final relation $\mathbf{y} \rightarrow \widetilde{\mathbf{y}}$. Applying this to the expression of $\widetilde{\mathbf{F}} \cdot \widetilde{\mathbf{F}}^{t}$ and solving for $\mathbf{B}$, we have: $\mathbf{B}=\widetilde{\mathbf{F}} \cdot \mathbf{P}^{t} \cdot \mathbf{F}^{-1}$. Substitution of $\mathbf{B}$ in $\mathbf{F} \cdot \widetilde{\mathbf{y}}=\mathbf{B} \cdot \mathbf{F} \cdot \mathbf{y}$ yields: $\widetilde{\mathbf{y}}=\mathbf{P}^{t} \cdot \mathbf{y}$.

The assessment of the $\mathbf{P}$ matrix in this context is known as the Orthogonal Procrustes problem (see [11] for details). The solution matrix is $\mathbf{P}=\mathbf{V} \cdot \mathbf{W}^{t}$, where $\mathbf{V} \cdot \mathbf{D} \cdot \mathbf{W}^{t}$ is the so-called Singular Value Decomposition of $\left(\mathbf{Y}^{t} \cdot \widetilde{\mathbf{Y}}\right) . \mathbf{Y}$ and $\widetilde{\mathbf{Y}}$ are $N \times D$ matrices formed by the vectors $\mathbf{y}$ and $\widetilde{\mathbf{y}}$ of the data point sets. $\mathbf{B}$ is obtained replacing $\mathbf{P}$ in $\mathbf{B}=\widetilde{\mathbf{F}} \cdot \mathbf{P}^{t} \cdot \mathbf{F}^{-1}$. Applying the Expectation Operator $(\mathbb{E})$ to $\widetilde{\mathbf{x}}=\mathbf{B} \cdot \mathbf{x}+\mathbf{t}$, we get $\mathbf{t}=\mathbb{E}\{\widetilde{\mathbf{x}}\}-\mathbf{B} \cdot \mathbb{E}\{\mathbf{x}\}$.

The matrix $\mathbf{A}$ in the particular affine transformation model can be obtained using the definition of the Moore-Penrose inverse. Following [3], for a $D \times N$ matrix $\mathbf{X}^{t}$ of points under some reference illumination condition, denote by $\widetilde{\mathbf{X}^{t}}$ the corresponding matrix when there is an illumination change. The matrix $\mathbf{A}$ that accomplishes: $\widetilde{\mathbf{X}^{t}} \approx \mathbf{A} \cdot \mathbf{X}^{t}$ is: $\mathrm{A}=\widetilde{\mathbf{X}^{t}} \cdot\left[\mathbf{X}^{t}\right]^{+} \cdot\left[\mathrm{X}^{t}\right]^{+}$is the Moore-Penrose inverse of matrix $\mathbf{X}^{t}$ (i. e., $\left[\mathbf{X}^{t}\right]^{+}=$ $\left.\mathbf{X} \cdot\left(\mathbf{X}^{t} \cdot \mathbf{X}\right)^{-1}\right)$. Considering [3]: $A_{i i}^{d}=\widetilde{X}_{i}^{t} \cdot\left[X_{i}^{t}\right]^{+}=\frac{\widetilde{X}_{i}^{t} \cdot X_{i}}{X_{i}^{t} \cdot X_{i}}$, where the single subscript $i$ denotes the $i$ th matrix row and the double subscript $i i$ denotes matrix element at row $i$ and column $i$.

\section{B Truncated Singular Value Decomposition technique.}

Let $\mathbf{F} \in \mathbb{R}^{m \times n}$ be a rectangular matrix with $m>n$. The Singular Value Decomposition $(S V D)$ of $\mathbf{F}$ is given by [12]: $\mathbf{F}=\mathbf{U} \cdot \boldsymbol{\Sigma} \cdot \mathbf{V}^{T}=\sum_{i=1}^{n} \mathbf{u}_{i} \sigma_{i} \mathbf{v}_{i}^{T}$, where $\mathbf{U}=\left(\mathbf{u}_{1}, \mathbf{u}_{2}, \ldots, \mathbf{u}_{n}\right)$ and $\mathbf{V}=\left(\mathbf{v}_{1}, \mathbf{v}_{2}, \ldots, \mathbf{v}_{n}\right)$, are orthonormal matrices, and where the numbers $\sigma_{i}$ are called the singular values of $\mathbf{F}$. If matrix $\mathbf{F}$ is ill-conditioned/rank deficient, the closest rank-k approximation $\mathbf{F}_{k}$ to $\mathbf{F}$ would be obtained by truncating the $S V D$ expansion at $k$ [12], i. e.: $\mathbf{F}_{k}=\sum_{i=1}^{k} \mathbf{u}_{i} \sigma_{i} \mathbf{v}_{i}^{T}, k \leq n$. Taking into account the properties of the orthonormal matrices $\mathbf{U}$ and $\mathbf{V}\left(\mathbf{U}^{-1}=\mathbf{U}^{T}, \mathbf{V}^{-1}=\mathbf{V}^{T}\right)$, the inverse matrix of $\mathbf{F}$ is: $\mathbf{F}^{-1}=\mathbf{V} \cdot \boldsymbol{\Sigma}^{-1} \cdot \mathbf{U}^{T}=\sum_{i=1}^{n} \mathbf{v}_{i} \cdot\left(\frac{1}{\sigma_{i}}\right) \cdot \mathbf{u}_{i}^{T}$, and and the closest rank-k approximation, $\left(\mathbf{F}^{-1}\right)_{k}$ of $\mathbf{F}^{-1}$ would be given by [12]: $\left(\mathbf{F}^{-1}\right)_{k}=\sum_{i=1}^{k} \mathbf{v}_{i} \cdot\left(\frac{1}{\sigma_{i}}\right) \cdot \mathbf{u}_{i}^{T}$. 


\section{References}

1. C. Y. Kuan, G. Healey, "Retrieving multispectral satellite images using physics-based invariant representations," IEEE Trans. on Pat. Analysis and Mach. Intel., vol. 18, (1996) 842-848.

2. C. Y. Kuan, G. Healey, "Using spatial filtering to improve spectral distribution invariants," Proc. SPIE, vol. 6233, (2006),62330G1-62330G12.

3. G. D. Finlayson, M. S. Drew, B. V. Funt, "Spectral sharpening: sensor transformations for improved color constancy," Journal of the Opt. Soc. of America, A, vol. 11, (1994) 1553-1563.

4. G. Wyszecki, W. S. Stiles, "Color Science: concepts and methods" Wiley, (2000).

5. R. Lenz, L. V. Tran, P. Meer, "Moment based normalization of color images," IEEE 3rd Workshop on Multimedia Signal Processing, (1998) 103-108.

6. G. Finlayson, S. S. Chatterjee, B. V. Funt, "Color Angular Indexing," Proc. 4th European Conference on Computer Vision, vol. 2 (1996) 16-27.

7. E. F. Vermote, D. Tanr, J. L. Deuz, M. Herman, J. J. Morcrette, "Second Simulation of the Satellite Signal in the Solar Spectrum: an overview," IEEE TGARS, vol. 35, (1997) 675-686.

8. "SEN2FLEX Data Acquisition Report" Project Contract No. 19187/05/I-EC (2005).

9. J. Heikkila, 'Pattern Matching with Affine Moment Descriptors," Pattern Recognition, vol. 37, (2004) 1825-1834.

10. J. Sprinzak, M. Werman, ”Affine Point Matching," Pat. Rec. Letters, vol. 15, (1994) 337-339.

11. P. H. Schonemann, "A generalized solution of the orthogonal Procrustes problem," Psychometrika, vol. 31, (1966) 1-10.

12. J. M. Varah, "On the numerical solution of ill-conditioned linear systems with applications to ill-posed problems,"SIAM Journal on Numerical Analysis, vol. 10, (1973), 257-267.

13. P. V. Espen, "Spectrum evaluation," Handbook of X-Ray Spectr. Marcel Dekker, (2001).

14. F. Gascon, M. Berger, "GMES Sentinel-2 Mission requirements document," T.R., European Space Agency, 2007.

15. S. Weiss. Measurement data deffinition and format description for MERIS. T.R. Astrium $\mathrm{GmbH}, 2001$.

16. Pedro Latorre Carmona, Reiner Lenz, Filiberto Pla, Jose M. Sotoca, ”Affine Illumination Compensation for Multispectral Images, " LNCS, 4522, (2007), 522-531.

17. Pedro Latorre Carmona, Jose E. Moreno, Filiberto Pla, Crystal B. Schaaf, " Affine Compensation of Illumination in Hyperspectral Remote Sensing Images," IEEE IGARSS, 2009. 